\title{
Increasing the scanning range of Lamb wave based SHM systems by optimizing the actuator-sensor design
}

\author{
C. Willberg $\cdot$ S. Duczek $\cdot$ U. Gabbert
}

Received: 11 April 2012/Revised: 16 November 2012/ Accepted: 13 December 2012

(C) Deutsches Zentrum für Luft- und Raumfahrt e.V. 2013

\begin{abstract}
Piezoelectric induced ultrasonic Lamb waves can easily be used for the development of structural health monitoring systems for aircraft and other thin-walled structures. The reduction of the excited wave amplitudes depends mainly on the traveling distance and the material damping, especially in composite materials which restricts the maximum scanning distance between a piezoelectric actuator and a sensor. But there are several possibilities to increase the scanning range of Lamb waves. In the present paper, the focus is on the influence of the adhesive layer and the resonances of the actuator to increase the amplitudes of the excited wave. The objective is to excite Lamb waves with higher amplitudes without increasing the electrical energy for the wave excitation. In the paper, a numerical optimization is proposed which aims at increasing the wave amplitudes by optimizing sensor parameters and the excitation frequency. It has been found that small changes in the geometry of the piezoelectric actuator patch and the use of an optimized excitation frequency elevate the amplitudes of waves significantly.
\end{abstract}

Keywords FEM - Optimization - Lamb wave - SHM · Adhesive layer

\footnotetext{
C. Willberg ( $\square)$

Institut für Faserverbundleichtbau und Adaptronik

Deutsches Zentrum für Luft und Raumfahrt (DLR),

38108 Braunschweig, Germany

e-mail: christian.willberg@dlr.de

S. Duczek - U. Gabbert

Institut für Mechanik, Otto-von-Guericke

Universität Magdeburg, Magdeburg, Germany

e-mail: sascha.duczek@st.ovgu.de

U. Gabbert

e-mail: ulrich.gabbert@ovgu.de
}

\section{Introduction}

The application of elastic guided waves to inspect structures has a long history, and is nowadays quite common for online monitoring of structures [6, 12]. In thin-walled structures, special elastic guided waves, named after Horace Lamb who has been the first to describe these waves in his famous article "On waves in an elastic plate" [18], occur. Such plate-like structures have free, parallel surfaces and are stress free at the top and bottom boundaries. Lamb wave based monitoring systems have several advantages compared to other non-destructive evaluation methods, e.g. the long range inspection capabilities and the high sensitivity to structural damages. Lamb waves exist in different mode types, a symmetric and an anti-symmetric one. For typical SHM applications, a frequency range is chosen where only the two basic modes appear [25]. The displacements in the cross section of a plate for stationary A0 and S0 modes are illustrated in Fig. 1.

To use Lamb waves efficiently, one important point is to understand the coupling effects between the actuator and the structure which affect the induced wave front. Detailed investigations about the adhesive layer thickness and shear stiffness are done by [3, 8, 23]. Ha et al. [13] performed investigations to understand the influence of ambient temperature, height and Young's modulus of the bonding layer on the emitted signal. The impact of soldering points at the top of a piezoelectric actuator has been shown by [26]. Huang et al. [16, 20] have demonstrated the effect of actuator resonances to the excited Lamb wave field.

Composites are one of the promising materials to build light-weight structures for several fields of application. They are widely used, e.g. for wind energy plants [9] and aerospace applications. But, unfortunately, the development, the design, the manufacturing, the safety inspections, 


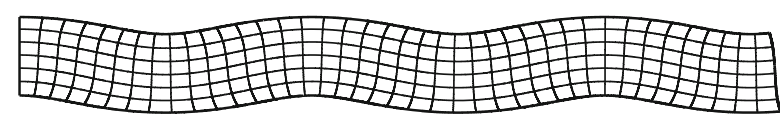

(a) A0 mode

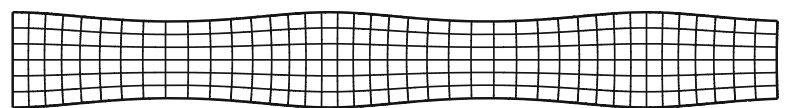

(b) S0 mode

Fig. 1 Lamb wave modes

Table 1 Example for difference in amplitude loss of the S0 and A0 mode [24]

\begin{tabular}{llllc}
\hline Mode & Material & $\begin{array}{l}\text { Frequency } \\
(\mathrm{kHz})\end{array}$ & $\begin{array}{l}\text { Attenuation } \\
\text { coefficient } \\
\left(\mathrm{mm}^{-1}\right)\end{array}$ & $\begin{array}{l}10 \% \text { amplitude } \\
\text { distance }(\mathrm{mm})\end{array}$ \\
\hline S0 & $\begin{array}{c}\text { CFRP woven } \\
\text { 8-ply }\end{array}$ & 250 & 0.0014 & 1,700 \\
A0 & $\begin{array}{c}\text { CFRP woven } \\
\text { 10-ply }\end{array}$ & 285 & 0.027 & 85 \\
& & & \\
\hline
\end{tabular}

the recycling, and others causes much more problems in comparison with isotropic metallic materials. If Lamb waves are applied for health monitoring systems, the higher damping characteristics of composites have to be taken into account. The different layers of composites cause mode interactions, mode reflections, and mode conversions [8]. Table 1 shows the amplitude loss in a plate of carbon fibre reinforced plastic (CFRP). It is important to note that the amplitude attenuation of the anti-symmetric mode is much higher in comparison to the symmetric mode. In the following at first, some brief information on the applied piezoelectric actuator materials and their modelling are given. As a first parameter, the influence of the adhesive layer on the excited Lamb wave amplitude is analyzed in detail. As a second parameter, the influence of the local actuator resonances is studied. Finally, the excitation frequency and the height and the length of an actuator patch are used as design parameters to maximize the amplitude of the excited Lamb wave for a given structure. Since the attenuation coefficient of the A0 mode is orders of magnitude higher than that of the S0 mode (Table 1), the S0 mode will be mainly regarded in the remaining paper. The results of the optimization are discussed and an outlook about further steps of investigations concludes the paper.

\section{Piezoelectric material}

The overall goal is to maximize the scanning range of the excited Lamb wave signal. Both excitation and sensing are

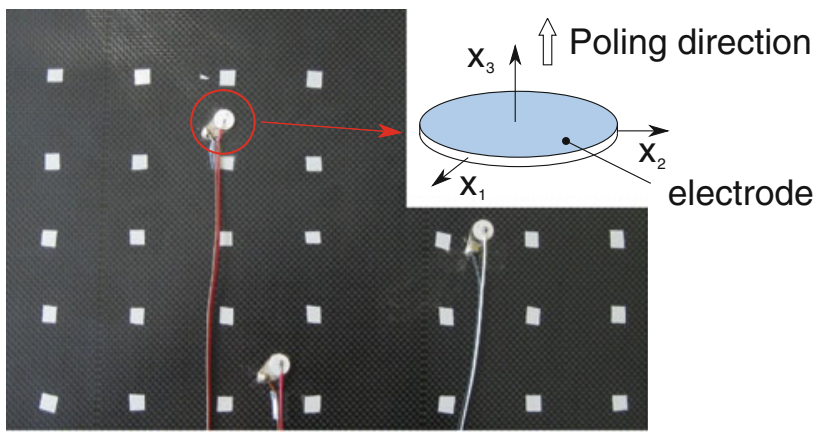

Fig. 2 Coordinates

performed using piezoelectric transducers (PZT). Piezoceramics are active materials which are able to transform mechanical energy into electrical energy (direct piezoelectric effect) and vice versa (converse piezoelectric effect). A piezoceramic transducer can be used as actuator and sensor alike. An example of applied piezoceramic actuators and their coordinate definition are given in (Fig. 2).

To implement the piezoelectric effect, a linearized material law can be used with sufficient accuracy in the small scale voltage range. Actuator applications are based on the converse piezoelectric effect, which is described by Eq. (1). The actuator is bonded to a structure and an external electric field $\mathbf{E}$ is applied, which results in an induced strain field $\boldsymbol{\varepsilon}$. Equation (2) illustrates the sensor effect. The dielectric displacement $\mathbf{D}$ is influenced by the electrical field as well as the mechanical stresses $\boldsymbol{\sigma}[7,19]$.

$\boldsymbol{\varepsilon}=\mathbf{S}^{E} \boldsymbol{\sigma}+\mathbf{d E}$

$\mathbf{D}=\mathbf{d}^{T} \boldsymbol{\sigma}+\mathbf{e}^{\sigma} \mathbf{E}$

The parameters $\mathbf{S}^{E}, \mathbf{d}$ and $\mathbf{e}^{\sigma}$ are the elastic compliance matrix measured at constant electric field $\mathbf{E}$, the piezoelectric coupling matrix and the permittivity matrix measured at constant mechanical stress $\sigma$, respectively. The coupled material law under the assumption of transversal isotropic material properties can be written as

$\left(\begin{array}{c}\varepsilon_{1} \\ \varepsilon_{2} \\ \varepsilon_{3} \\ \varepsilon_{4} \\ \varepsilon_{5} \\ \varepsilon_{6} \\ \hline D_{1} \\ D_{2} \\ D_{3}\end{array}\right)=\left(\begin{array}{cccccc|ccc}s_{11}^{E} & s_{12}^{E} & s_{13}^{E} & 0 & 0 & 0 & 0 & 0 & d_{31} \\ s_{12}^{E} & s_{11}^{E} & s_{13}^{E} & 0 & 0 & 0 & 0 & 0 & d_{31} \\ s_{13}^{E} & s_{13}^{E} & s_{33}^{E} & 0 & 0 & 0 & 0 & 0 & d_{33} \\ 0 & 0 & 0 & s_{44}^{E} & 0 & 0 & 0 & d_{15} & 0 \\ 0 & 0 & 0 & 0 & s_{44}^{E} & 0 & d_{15} & 0 & 0 \\ 0 & 0 & 0 & 0 & 0 & s_{66}^{E} & 0 & 0 & 0 \\ \hline 0 & 0 & 0 & 0 & d_{15} & 0 & e_{11}^{\sigma} & 0 & 0 \\ 0 & 0 & 0 & d_{15} & 0 & 0 & 0 & e_{11}^{\sigma} & 0 \\ d_{31} & d_{31} & d_{33} & 0 & 0 & 0 & 0 & 0 & e_{33}^{\sigma}\end{array}\right) \cdot\left(\begin{array}{c}\sigma_{1} \\ \sigma_{2} \\ \sigma_{3} \\ \sigma_{4} \\ \sigma_{5} \\ \sigma_{6} \\ \hline E_{1} \\ E_{2} \\ E_{3}\end{array}\right)$

with $s_{66}^{E}=2\left(s_{11}^{E}-s_{12}^{E}\right)$ as shear modulus belonging to the plane of isotropy. Using the piezoceramic as a sensor, the conversion of mechanical strain to a voltage output can be derived. The charge $q$ and the voltage $V_{\mathrm{C}}$ generated across the sensor electrodes are related by the capacitance $C_{p}$ of the sensor as 
$V_{\mathrm{C}}=\frac{q}{C_{p}}$.

In further investigations, a two-dimensional model is considered. In this case, the electric displacement is related to the generated charge by the expression

$q=\int D_{3} d A_{3}=\int D_{3} b_{C} \mathrm{~d} x_{1}$.

According to [23], a piezoelectric sheet can be treated as a parallel plate capacitor, with a capacitance given by

$C_{p}=\frac{e_{33}^{\sigma} \cdot l_{C} \cdot b_{C}}{t_{C}}$.

$e_{33}^{\sigma}$ describes the dielectric permittivity, $t_{C}$ is the height, $l_{C}$ is the length, and $b_{C}$ is the width of the piezoceramic sensor. In the sensor case, the electrical field $\mathbf{E}$ is zero inside a piezoelectric sensor $[8,23]$ which results in an electric displacement

$D_{3}=\left[\begin{array}{lll}d_{31} & d_{33} & 0\end{array}\right] \cdot\left[\begin{array}{l}\sigma_{1} \\ \sigma_{3} \\ \sigma_{4}\end{array}\right]$.

In piezoceramic patches metallized on top and bottom, the voltage can be calculated directly from the electrical flux $D_{3}$ using the Eqs. (4)-(6). Note that shear stress in the 1-2 plane, $\sigma_{6}$, is not capable of generating any electric response

$V_{0}=\frac{q}{C_{p}}=\frac{t_{C}}{e_{33}^{\sigma} \cdot l_{C}} \cdot \int D_{3} \mathrm{~d} x_{1}$.

\section{Adhesive layer influence}

If a patch actuator is glued to the surface of a structure, a shear lag effect can be observed. The shear lag is caused by an adhesive layer of finite thickness between the actuator and the host structure (Fig. 3). The excitation signal from the actuator is mainly transmitted to the structure through interfacial shear stresses within the bonding layer [12]. The shear lag causes a reduction of shear strain transfer between the PZT actuator and the host structure. With a lower shear modulus and a thicker adhesive layer, the shear lag effect becomes more pronounced. As a result, the signal amplitude is significantly reduced [13] if the resonance effects play a minor role (valid for thin piezoelectric wafer

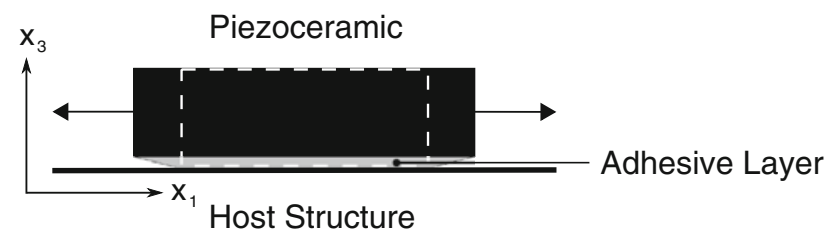

Fig. 3 Schematic representation of the shear lag effect

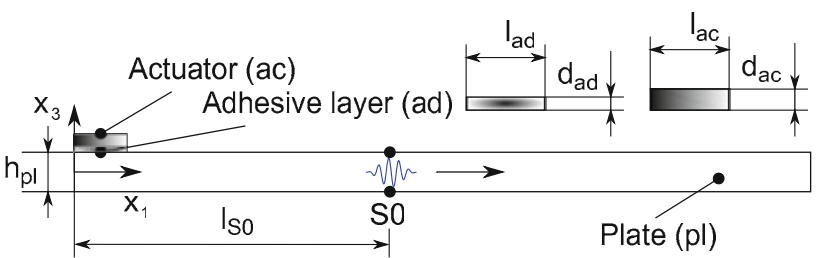

Fig. 4 Two-dimensional symmetric model used to investigate adhesive layer effects (actuator: PZT- $5 \mathrm{H}, l_{\mathrm{ac}}=3.35 \mathrm{~mm}, d_{\mathrm{ac}}=0.23 \mathrm{~mm}$; adhesive layer: paraffin wax $, l_{\mathrm{ad}}=3.35 \mathrm{~mm}, d_{\mathrm{ad}}=50 \mu \mathrm{m}$ plate: aluminium, $l_{p l}=200 \mathrm{~mm}, h_{p l}=2 \mathrm{~mm}$ ); the results are being measured at a point at a distance of $l S 0=100 \mathrm{~mm}$ from the actuator

active sensors-PWAS). Due to this effect, the effective length of a piezoelectric actuator is smaller than the given length. Consequently, it can be assumed that only a fraction of the actual surface of a piezoelectric actuator contributes to the strain transfer [8]. Thus, correction factors have to be applied to account for the shear lag effect. Based on analytical models (e.g. beam theory), [10], [12] and [23] derived correction factors for various problems assuming the application of thin piezoceramics $(\approx 0.2 \mathrm{~mm})$ only.

To investigate the influence of an adhesive layer, we use a numerical approach. The two-dimensional model for a finite element analysis including its dimensions is shown in Fig. 4. The $x_{1}$ component of the displacement field is constrained to be zero at the origin (symmetry boundary condition). Thus, we have to model only one half of the symmetric domain. All other boundaries are free. To ensure high accuracy results, the mesh parameters are chosen so that the largest element size $\left(L_{\mathrm{e}}\right)$ is substantially smaller than the shortest wavelength $\left(\lambda_{\min }\right)$ [2]

$L_{\mathrm{e}} \leq \frac{\lambda_{\min }}{20}$.

The simulations are performed utilizing the software package ABAQUS version 6.7-1 (element type: CPE4R). We are convinced that all essential effects caused by a bonding layer can be investigated with two-dimensional finite element models, based on the assumption of a plain strain state, such assuming an infinite plate in $x_{3}$ direction. Therefore, computationally very costly three-dimensional simulations can be avoided. The material properties of the model are given in (Table 2).

Table 2 Material properties of aluminium, paraffin wax and PZT-5H

\begin{tabular}{llll}
\hline Parameter & Aluminum & Paraffin wax [27] & PZT-5H [23] \\
\hline$E(\mathrm{GPa})$ & 70 & 1.02 & 174 \\
$v(-)$ & 0.33 & 0.37 & 0.3 \\
$d_{31}(p C / N)$ & - & - & -274 \\
$d_{33}(p C / N)$ & - & - & 593 \\
$e_{33}^{\sigma}(n F / m)$ & - & - & 30.1 \\
\hline
\end{tabular}


The time-dependent excitation is given by

$A(t)=\left\{\begin{array}{cc}\sin (\omega t) \sin ^{2}\left(\frac{\omega t}{2 n}\right), & t<\frac{2 n \pi}{\omega}=\frac{n}{f} \\ 0, & t \geq \frac{n}{f}\end{array}\right.$

Choosing $n=5$ yields a smooth 5 -cycle sinusoidal burst windowed by a Hann-window with the center frequency $f$. We will report on the results of three different frequencies $(100,200,355 \mathrm{kHz})$. In this frequency range only the S0- and A0-mode exist for the given plate (plate thickness of $2 \mathrm{~mm}$ ). To analyze the signal properly, it is being decomposed into its two components. To separate the SO from the A0 signal component, the following equations are used

$u_{1_{S 0}}=\frac{1}{2}\left(u_{1_{\text {top }}}+u_{1_{\text {bot }}}\right)$,

$u_{3_{S 0}}=\frac{1}{2}\left(u_{3_{\mathrm{top}}}-u_{3_{\mathrm{bot}}}\right)$.

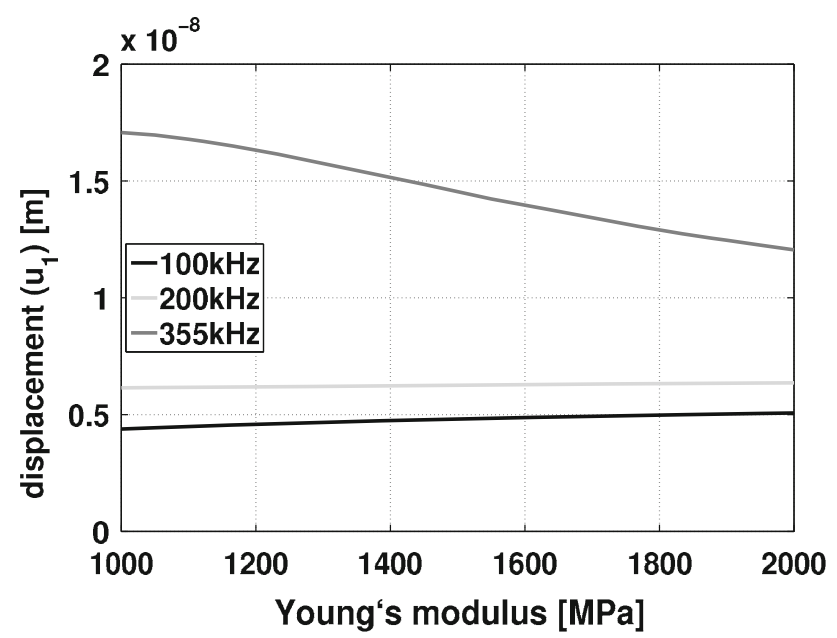

(a)

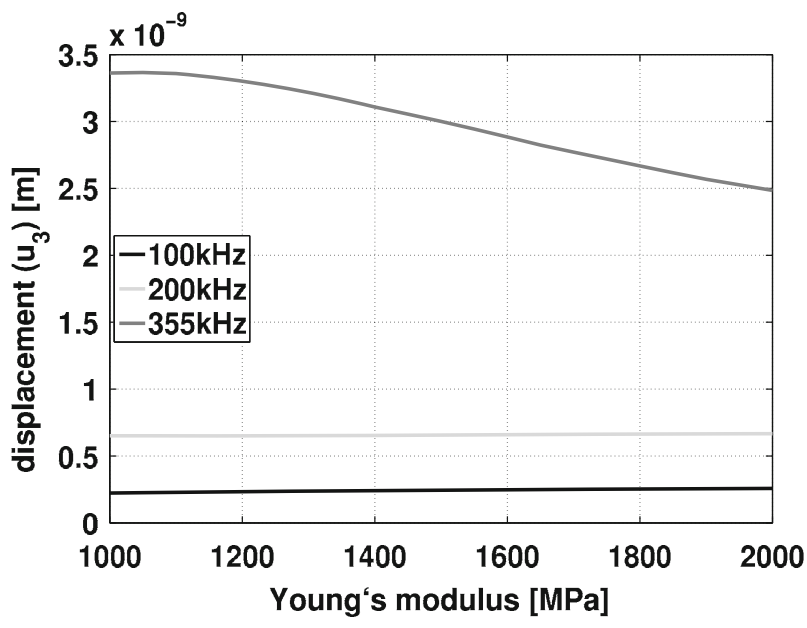

(b)

Fig. 5 Relation between wave amplitude and Young's modulus for S0. a $u_{1}$ displacement, $\mathbf{b} u_{3}$ displacement
Figures 5 and 6 show the highest value of the displacement field at the measurement point. It is ensured that the analyzed signals do not contain any reflected signals and are not interfered by other disturbances. Figure 5 shows the influence of the Young's modulus of the adhesive layer $\left(E_{\mathrm{ad}}=1 \mathrm{GPa} . .2 \mathrm{GPa}\right)$ to the maximal wave amplitude (S0) in $x_{1}$ direction (Fig. 5a) and $x_{3}$ direction (Fig. 5b), respectively. These results are obtained using a constant adhesive layer thickness of $d_{\mathrm{ad}}=50 \mu \mathrm{m}$. According to [15], the change in the Young's modulus is equivalent to a change in the ambient temperature. A temperature range from -20 to $40^{\circ} \mathrm{C}$ can be modelled by the chosen variation $E_{\text {ad }}$. The results show a slight, monotonic amplitude increase with increasing Young's modulus at 100 and 200 $\mathrm{kHz}$, which agrees with the shear lag effect model [23]. At $355 \mathrm{kHz}$, the amplitude signal is more heavily affected by the stiffness of the bonding layer (see Fig. 5). The reason is

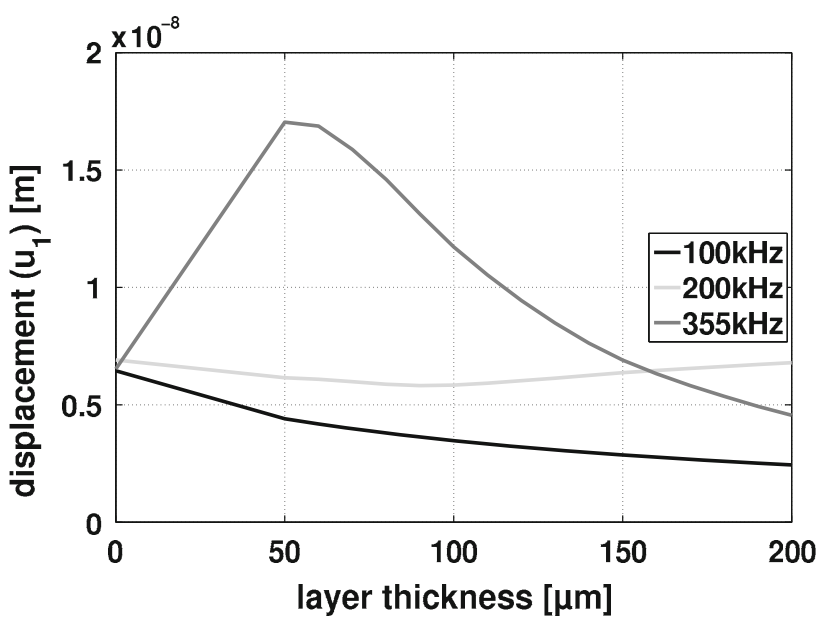

(a)

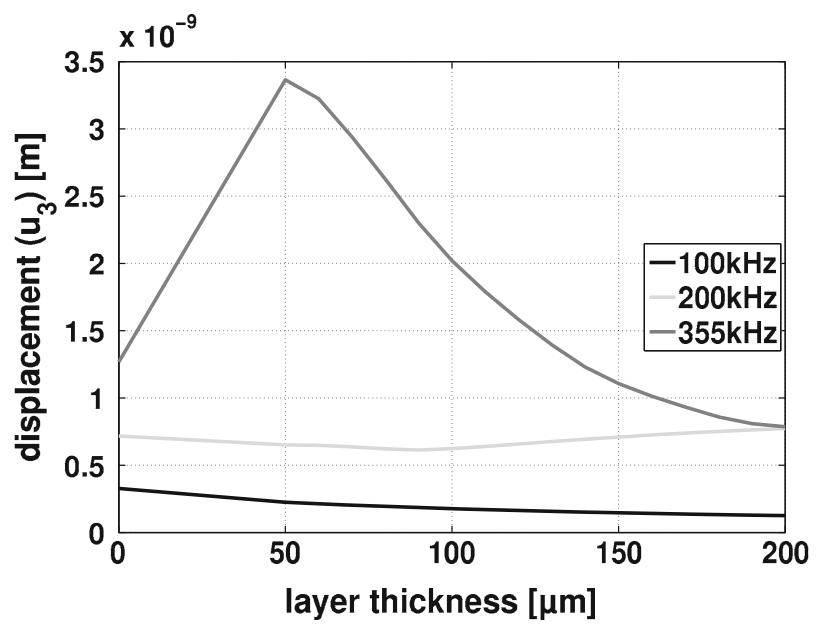

(b)

Fig. 6 Relation between wave amplitude and thickness of the adhesive layer for $\mathrm{S} 0$. a $u_{1}$ displacement, $\mathbf{b} u_{3}$ displacement 
that the frequency of $355 \mathrm{kHz}$ coincides with the resonance frequency of the first radial dominant actuator eigenmode of the structure under investigation $\left(E_{\mathrm{ad}}=1 \mathrm{GPa}\right)$. Hence, the highest signal amplitude is to be expected if $f=355$ $\mathrm{kHz}$. The increasing Young's modulus shifts the resonance frequency of the coupled actuator-plate system to higher frequencies. Consequently, the signal amplitude decreases monotonically. Even for a higher Young's modulus, the system is still close to a resonance frequency and, thus, an increased signal amplitude is measured at the sensor. At $355 \mathrm{kHz}$, the resonance effect becomes dominant and superimposes the shear lag effect. It is obvious that the excited amplitudes of the Lamb waves can remarkably improved using such resonance effects.

Figure 6 demonstrates the influence of the thickness of the bonding layer to the wave amplitude (S0) in $x_{1}$ direction (Fig. 6a) and $x_{3}$ direction (Fig. 6b), respectively. The simulations have been executed using a constant Young's modulus $E_{\text {ad }}=1.02 \mathrm{GPa}$. At $100 \mathrm{kHz}$, the amplitude decreases monotonically as the adhesive layer thickness increases due to the dominance of the shear lag effect. Different effects can be perceived at 200 and $355 \mathrm{kHz}$. Again, a shift in the resonance frequency is responsible for the differing behavior of the coupled system for various excitation frequencies. With larger adhesive layer thickness, the coupled actuator-plate system becomes softer. Accordingly, the resonance shifts to lower frequencies. Depending on the eigenfrequency of the structure either the resonance or the shear lag effect dominates the overall behavior. Thus, it is possible that the resonance effect compensates the shear lag effect and results in higher amplitudes even for higher bonding layer thicknesses. Both Fig. $6 a, b$ shows a maximum wave amplitude at an adhesive layer thickness of $d_{\mathrm{ad}}=50 \mu \mathrm{m}$. As mentioned before for $d_{\mathrm{ad}}=50 \mu \mathrm{m}$ and $E_{\text {ad }}=1.02 \mathrm{GPa}$ a resonance frequency occurs at exactly $355 \mathrm{kHz}$.

That means the amplitude signal is governed by two opposing effects:

1. shear lag effect,

2. resonance effect.

Whether the one or the other effect is dominating in the received signal depends on the excitation frequency and the adhesive layer thickness. Ha [14] has shown that the shear lag effect plays a minor role in the high frequency domain. The adhesive layer parameters shift the resonance frequency of the coupled actuator-structure system. This phenomenon can be seen in Fig. 7. With increasing bonding layer thickness, the resonance frequency decreases since the coupled actuator-structure system becomes globally softer.

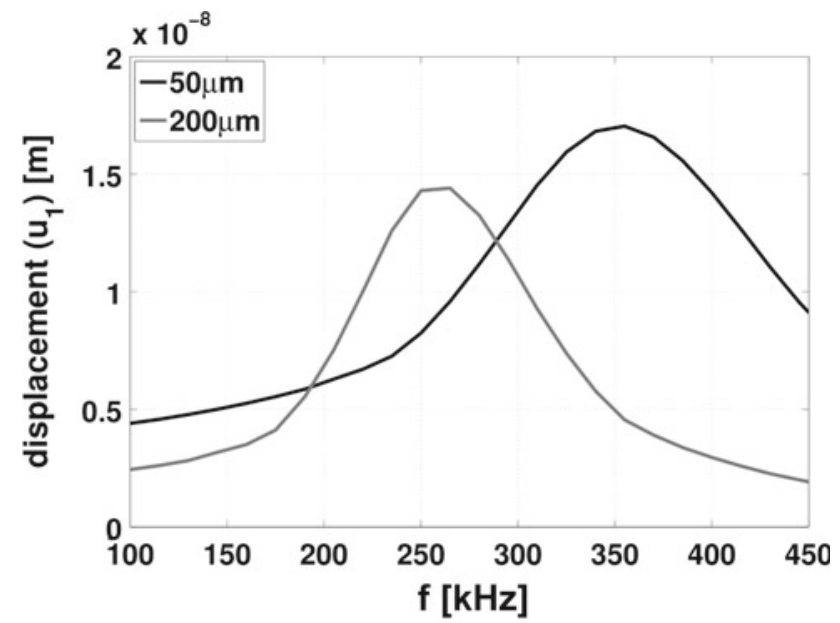

(a)

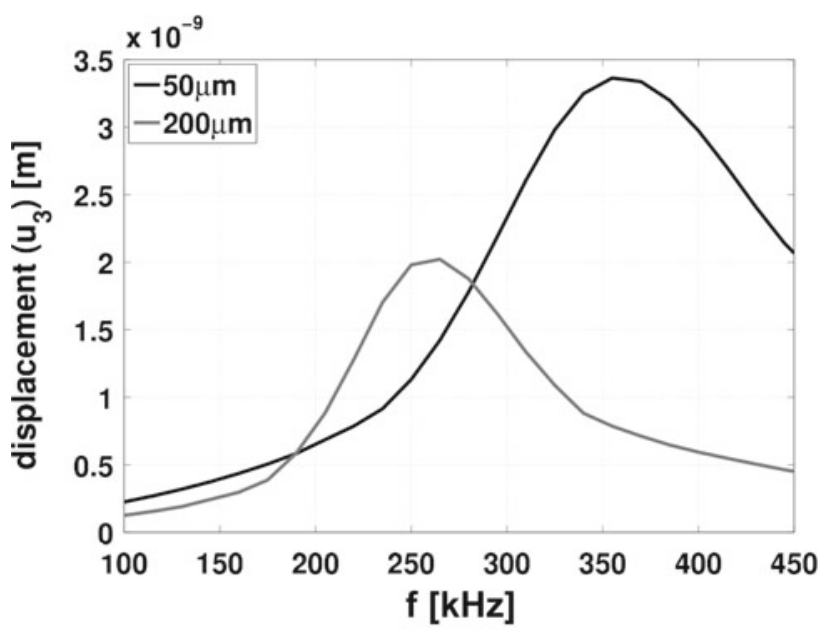

(b)

Fig. 7 Signal amplitude for various bonding layer thicknesses (S0). a $u_{1}$ displacement, $\mathbf{b} u_{3}$ displacement

Table 3 Optimal frequencies for Lamb mode generation in a CFRP plate up to $500 \mathrm{kHz}(d=2 \mathrm{~mm})$

\begin{tabular}{llll}
\hline$n$ & $\lambda(\mathrm{m})$ & $f$ of A0 $(\mathrm{kHz})$ PMMA & $f$ of S0 $(\mathrm{kHz})$ \\
\hline 0 & 0.0200 & 40.7 & 276 \\
1 & 0.0067 & 186 & $>500$ \\
2 & 0.0040 & 335 & $>500$ \\
3 & 0.0029 & 480 & $>500$ \\
\hline
\end{tabular}

\section{Resonance effects}

The design of a piezoceramic patch actuator for structural health monitoring applications should guarantee a most effective Lamb wave generation. According to [11], [17], and [24], the most effective generation of Lamb waves is accomplished, if the actuator length or diameter $d$, 
respectively, is related to the wavelength in the following manner

$d=\lambda(n+0.5)$.

$\lambda$ describes the wavelength and $n$ is an arbitrary integer $(n=0,1,2, \ldots)$. To investigate the resonance effects, a CFRP plate with a $\left[(0 / 90)_{f} /+45 /-45 / \overline{(0 / 90)}_{f}\right]_{S}$ layup and a plate thickness of $2 \mathrm{~mm}$ is examined. Table 3 shows the first four optimal wavelengths.

The experimental results are displayed in Fig. 8a-c. In addition to two-dimensional scans of the out-of-plane displacements with a PSV-300 laser scanning vibrometer [20], several piezoceramic actuators are measured using a 3D-laser vibrometer (PSV 400 3D). The different curves from Fig. 8 show a close relation between the resonances of the piezoelectric actuators and the spectra of the Lamb wave modes. The frequencies corresponding to certain values of the wavelength of Lamb waves for the CFRP plate have been derived from Eq. (13) and are given in Table 3 for frequencies up to $500 \mathrm{kHz}$ both for the $\mathrm{S} 0$ and A0 mode. Comparing the measured resonance frequencies with the calculated data from Table 3 , it can concluded that Eq. (13) cannot be applied for thicker piezoceramic actuators. It is evident that the eigenfrequencies of the actuator in connection with the structure have a major influence on the excited Lamb wave amplitudes. The assumption that an optimal excitation of Lamb modes occurs if the diameter of the piezoceramic disk is one half of an integer number of the wavelengths does not hold in any circumstance [20]. Only for thin ceramics it can be confirmed that the lowest eigenfrequency calculated with Eq. (13) coincides with the measurements. In all other cases, no agreement with measurements could be observed, meaning that the application of Eq. (13) to estimate optimal excitation frequencies cannot be recommended.

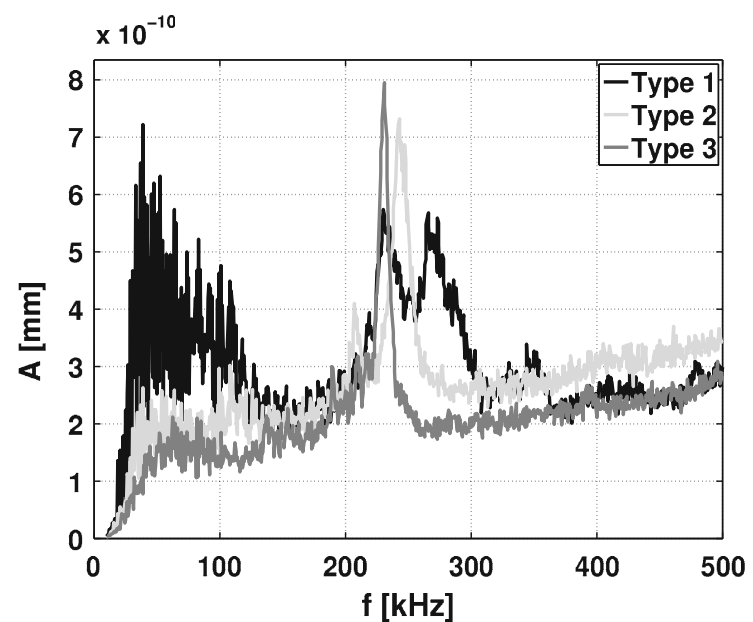

(a)

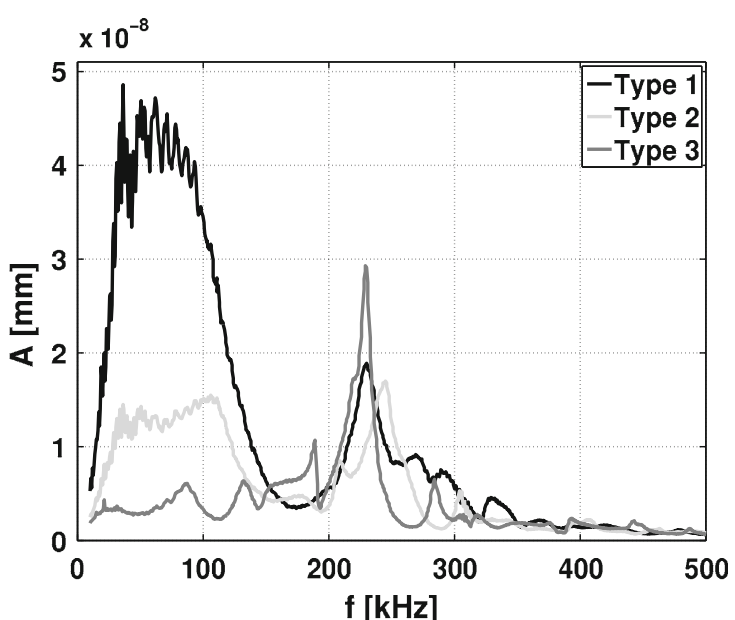

(b)

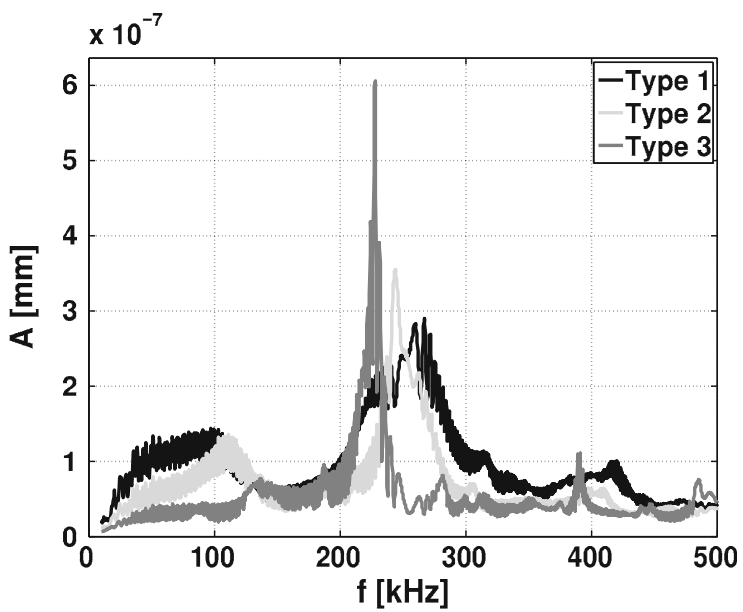

(c)

Fig. 8 Type $1(d=10 \mathrm{~mm}, h=0.5 \mathrm{~mm})$, Type $2(d=10 \mathrm{~mm}, h=1 \mathrm{~mm})$ and Type $3(d=10 \mathrm{~mm}, h=2 \mathrm{~mm})$ for a CFRP plate. a S0 spectra, b A0 spectra, c spectra of piezoceramic discs 
For SHM systems, the application of higher frequencies is preferable due to an improved resolution and a better ability to detect damages. The resonances play a major role in the excitation of Lamb waves. For thicker piezoceramics, it is the dominant effect [14] which influences the amplitude of the excited Lamb waves. Figure 7 a shows a frequency shift which is caused by a change in the adhesive layer thickness. The amplitude change in $u_{1}$ direction is relatively small, since the piezoceramic patch sensor primarily works with the $d_{31}$ effect. Thus, only a change of the $u_{1}$ displacement changes the measurable sensor voltage considerably. As shown before, the shear lag effect plays a minor role near a resonance frequency.

\section{Optimization model}

To increase the scanning range of S0 Lamb waves, several design parameters could be included into the optimization process. In the following study, the sensor height, the sensor length, and the frequency are included in the vector $\mathbf{x}$ of design parameters. Here, a fixed actuator geometry is assumed to gain a better understanding of the impact exerted by a frequency variation during the optimization process. As objective function, the maximal amplitude of the sensor voltage is used, which can be written as

$\max _{x \in S} f(\mathbf{x}), \quad S=\mathbb{R}^{N}$

The optimization method being used is the line-evolution strategy (L-ES) method proposed by [4]. It is a modified evolutional strategy (ES) which takes the knowledge from previous iteration steps into account, to increase the convergence rate of this method. For more details, we refer to $[4,5]$; see also $[1,21,22]$. The geometry of the applied test models is given in Fig. 9a. Two actuators are used to separate the modes. If the upper actuator vibrates in the

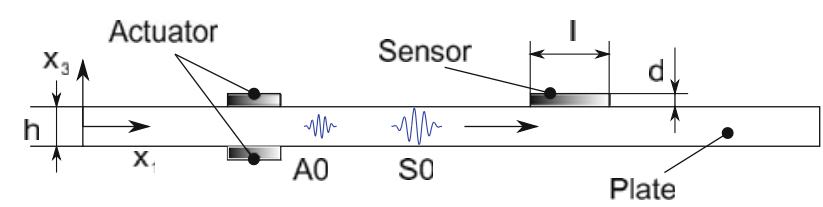

(a)

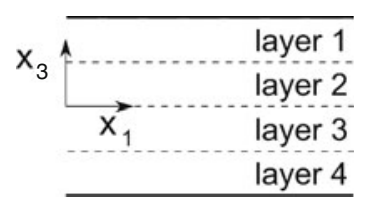

(b)

Fig. 9 Two-dimensional model for optimization. a Geometry of the optimization model (length $=10 \mathrm{~mm}$, height $=1 \mathrm{~mm}$, PIC-181). b Layer setup of the plate $\left(h_{\text {layer }}=0.5 \mathrm{~mm}\right)$ same phase like the lower one, the symmetric S0 mode is dominantly excited. If both actuators are driven with a $180^{\circ}$ phase shift the amplitude of the anti-symmetric A0 mode increases [26]. As already mentioned, the central frequency $f$ of an excited Hann-window signal (Eq. 10), the height $d$ and the length $l$ of the sensor are the design parameters to be optimized. The maximization of the voltage output at the piezoceramic sensor is used as objective function. The adhesive layer thickness is not varied here since it causes a shift in the resonance frequency (Fig. 7) only, which has no influence on the optimization results.

Layered plates with different material properties are investigated to include also the influence of the material properties in the study. In Table 4, the material properties of the plates under investigation are given. The layer setup is illustrated in Fig. 9b. We start with an isotropic aluminium plate and proceed to composites with different material properties of the layers. The material parameters of the piezoceramic (PIC-181) are given in [20]. As density

Table 4 Material properties of a four layer laminate with a thickness $(h=2 \mathrm{~mm})$ for the different types of optimization problems

\begin{tabular}{cllll}
\hline Parameter & MOD1 & MOD2 & MOD3 & MOD4 \\
\hline Layer 1 & & & & \\
$E_{1}(\mathrm{GPa})$ & 70 & 174 & 174 & 66.9 \\
$E_{3}(\mathrm{GPa})$ & 70 & 66.9 & 66.9 & 66.9 \\
$G_{13}(\mathrm{GPa})$ & 26.31 & 24 & 24 & 24 \\
$v_{13}(-)$ & 0.33 & 0.414 & 0.414 & 0.3 \\
Layer 2 & & & & \\
$E_{1}(\mathrm{GPa})$ & 70 & 174 & 66.9 & 174 \\
$E_{3}(\mathrm{GPa})$ & 70 & 66.9 & 66.9 & 66.9 \\
$G_{13}(\mathrm{GPa})$ & 26.31 & 24 & 24 & 24 \\
$v_{13}(-)$ & 0.33 & 0.414 & 0.3 & 0.414 \\
Layer 3 & & & & \\
$E_{1}(\mathrm{GPa})$ & 70 & 174 & 66.9 & 174 \\
$E_{3}(\mathrm{GPa})$ & 70 & 66.9 & 66.9 & 66.9 \\
$G_{13}(\mathrm{GPa})$ & 26.31 & 24 & 24 & 24 \\
$v_{13}(-)$ & 0.33 & 0.414 & 0.3 & 0.414 \\
Layer 4 & & & & \\
$E_{1}(\mathrm{GPa})$ & 70 & 174 & 174 & 66.9 \\
$E_{3}(\mathrm{GPa})$ & 70 & 66.9 & 66.9 & 66.9 \\
$G_{13}(\mathrm{GPa})$ & 26.31 & 24 & 24 & 24 \\
$v_{13}(-)$ & 0.33 & 0.414 & 0.414 & 0.3 \\
\hline
\end{tabular}

Table 5 Parameters for the optimization and the range of variation

\begin{tabular}{llll}
\hline Parameter & Start value & Lower border & Upper border \\
\hline Frequency $(\mathrm{kHz})$ & 120 & 100 & 400 \\
Sensor height $(\mathrm{mm})$ & 1.0 & 0.2 & 2.5 \\
Sensor length $(\mathrm{mm})$ & 10.0 & 2.0 & 25.0 \\
\hline
\end{tabular}


of the model 1 (MOD1), aluminum with $\rho=2700 \mathrm{~kg} / \mathrm{m}^{3}$ is used. For models 2-4 (MOD2-MOD4), CFRP with a volume fraction of $60 \%$ is chosen. The homogenized density is given as $\rho=1396 \mathrm{~kg} / \mathrm{m}^{3}$. The adhesive layer between the piezoceramics and the plate is neglected in this study. Table 5 shows the initial values of the design parameters and the range in which they are varied. The central frequency range is chosen so that only the two basic modes, the S0 and A0 Lamb wave mode, exist.

\section{Results and discussion}

In the following, the main results of the parameter study are presented and discussed. Table 6 shows the converged (optimized) solution marked as "best" one in comparison to the "worst" solution that occurred during the optimization process. In general, it can be noted that the amplitudes of the optimized models are significantly higher than those of the "worst" solutions. Furthermore, it can be
Table 6 Worst and best parameters of the optimization process

\begin{tabular}{|c|c|c|c|c|c|c|c|c|}
\hline \multirow[t]{2}{*}{ Parameter } & \multicolumn{2}{|l|}{ MOD1 } & \multicolumn{2}{|l|}{ MOD2 } & \multicolumn{2}{|l|}{ MOD3 } & \multicolumn{2}{|l|}{ MOD4 } \\
\hline & Worst & Best & Worst & Best & Worst & Best & Worst & Best \\
\hline Frequency $(\mathrm{kHz})$ & 251 & 320 & 110 & 351 & 200 & 228 & 377 & 182 \\
\hline Sensor height (mm) & 0.5 & 2.4 & 0.5 & 2.3 & 1.5 & 1.9 & 0.6 & 1.9 \\
\hline Sensor length (mm) & 24.7 & 4.2 & 7.1 & 3.6 & 21 & 10.3 & 12.6 & 4.2 \\
\hline
\end{tabular}

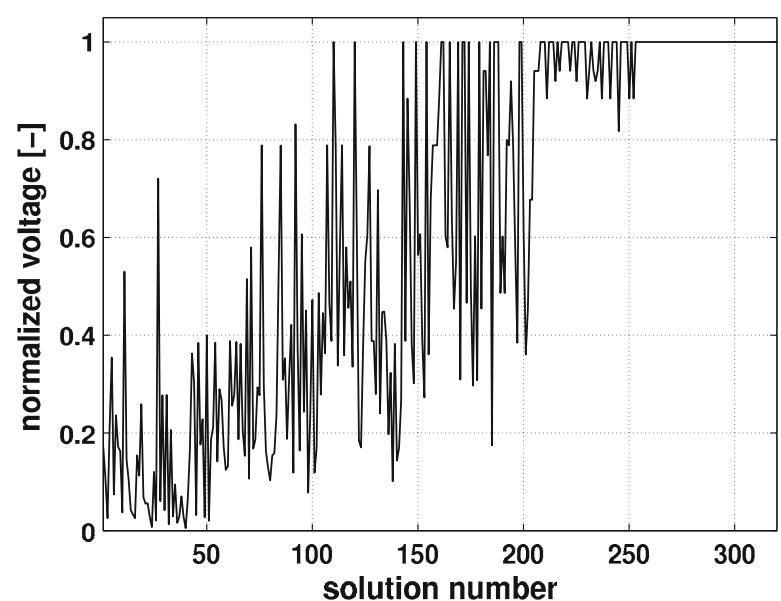

(a)

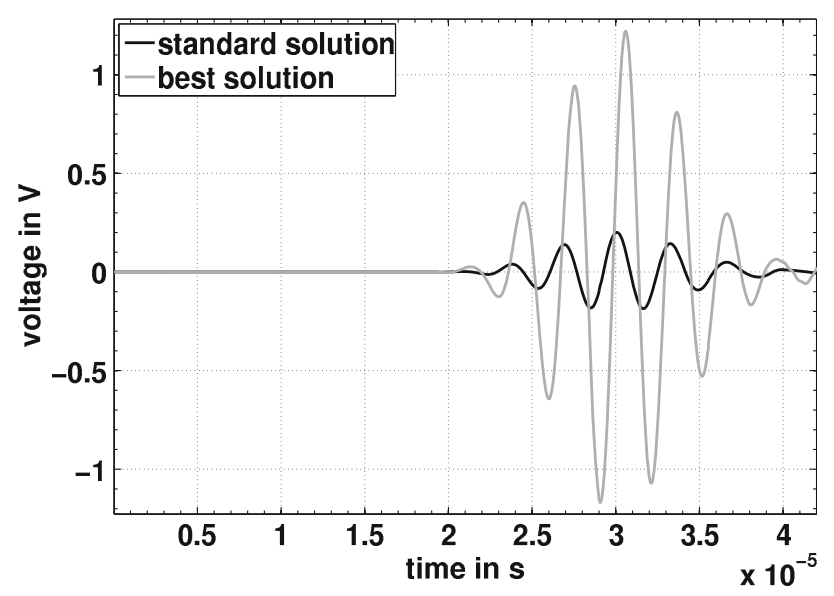

(b)

Fig. 10 Results of MOD1. a Convergence process of the optimization. b Comparison of the sensor signal of the standard and best solution

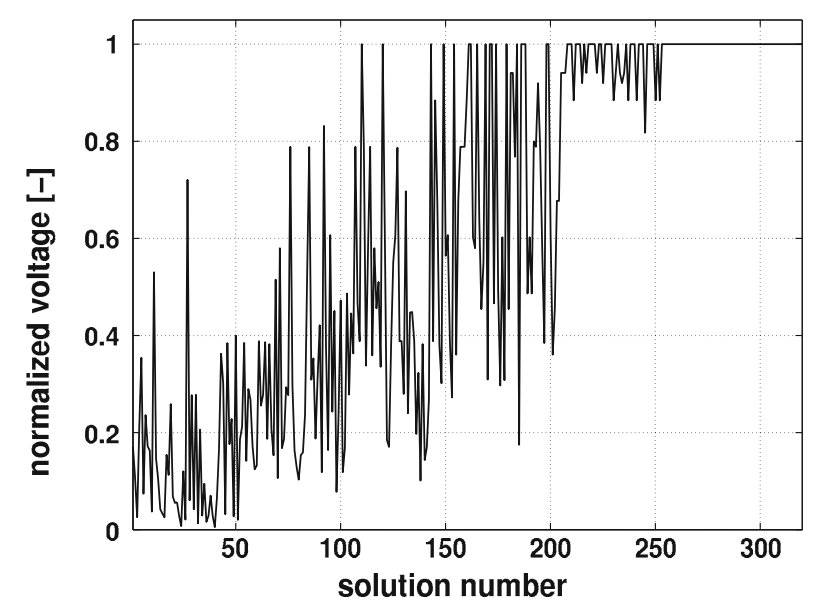

(a)

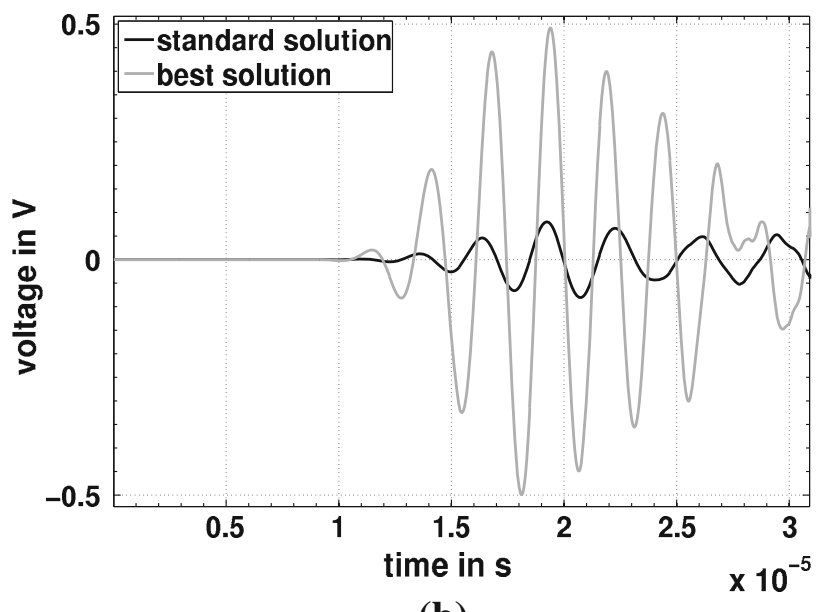

(b)

Fig. 11 Results of MOD2. a Convergence process of the optimization. b Comparison of the sensor signal of the standard and best solution 


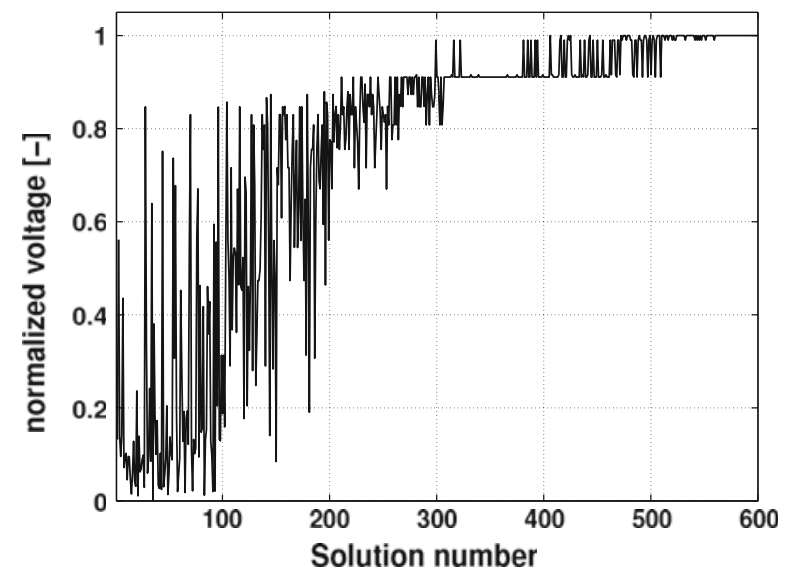

(a)

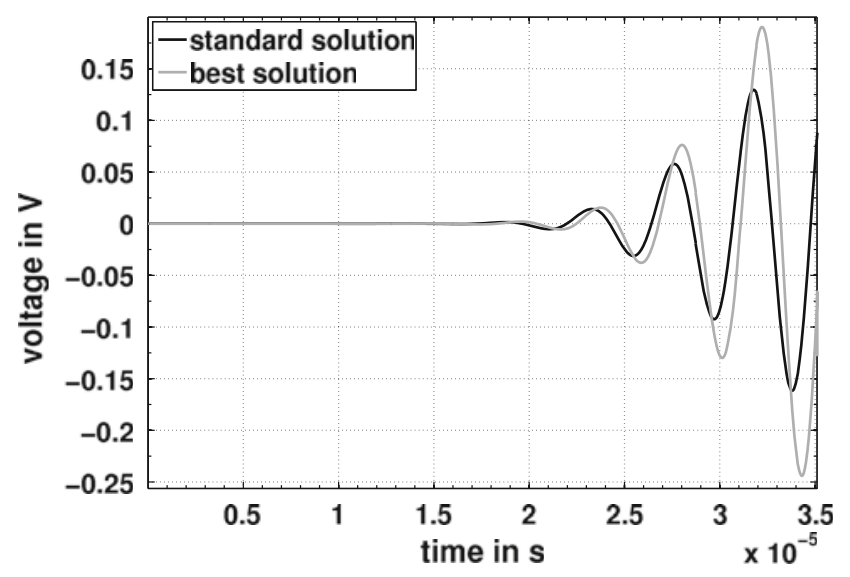

(b)

Fig. 12 Results of MOD3. a Convergence process of the optimization. b Comparison of the sensor signal of the standard and best solution

stated that the thickness of the sensor tends to higher and the length to lower values. These observations agree quite well with the general expectations arising from Eq. (8).

Figures 10, 11, 12 and 13 illustrate the results of the optimization procedure. Figures 10a, 11, 12 and 13a display the convergence process. The voltage amplitude is normalized with respect to the highest amplitude seen during the optimization. If the optimal solution is reached the variation of the results tends towards zero and thus the normalized amplitude is equal to one. Figures 10b, 11, 12 and $13 \mathrm{~b}$ plot the signal at the sensor. The signals received with the optimized sensor geometry ("best") are compared with the voltage signal of a piezoceramic sensor with the same geometry as the applied actuator $(d=10 \mathrm{~mm}, h=1$ $\mathrm{mm}$ ). In the graphs (Figs. 10b, 11, 12, 13b), this solution is named as "standard" solution. The excitation frequency of both the "standard" and the "best" sensor geometry corresponds to the optimized frequency (Table 6). All curves convey a strong elevation in the voltage output of the

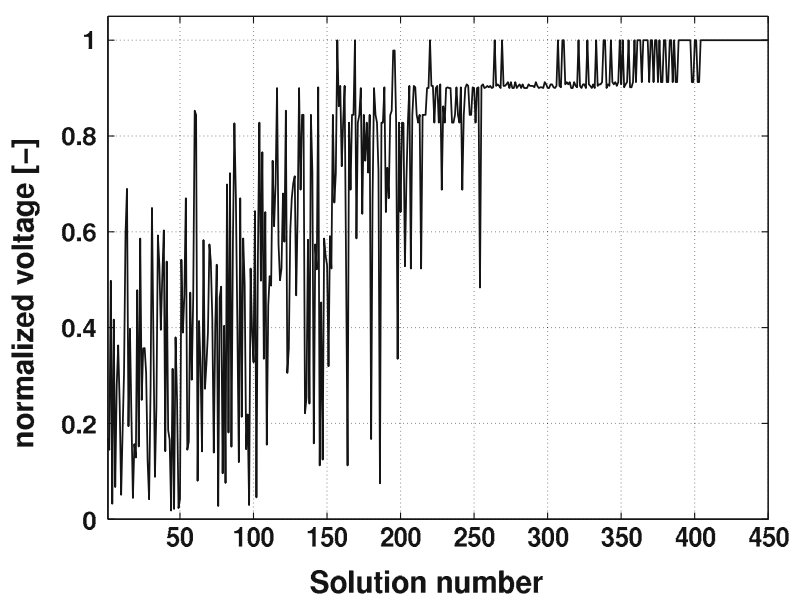

(a)

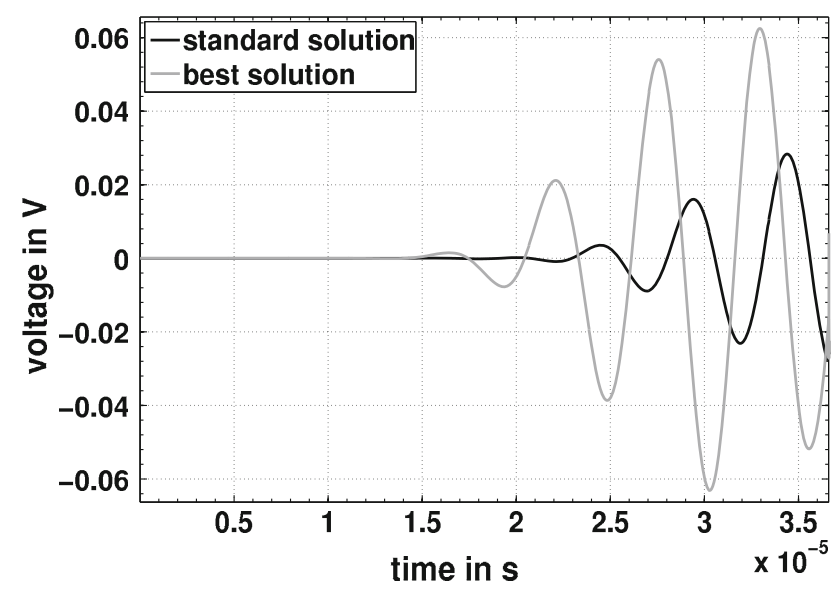

(b)

Fig. 13 Results of MOD4. a Convergence process of the optimization. b Comparison of the sensor signal of the standard and best solution

Table 7 Comparison of the optimization results with the optimal wavelength and the resonance

\begin{tabular}{lllcl}
\hline Model & EF $(\mathrm{kHz})$ & EW $(\mathrm{mm})$ & OW $(\mathrm{mm})$ & RF $(\mathrm{kHz})$ \\
\hline MOD1 & 320 & 8.2 & 8.4 & - \\
MOD2 & 351 & 15.9 & 7.2 & 300 \\
MOD3 & 228 & 20.8 & 20.6 & 230 \\
MOD4 & 182 & 26.4 & 8.4 & 180 \\
\hline
\end{tabular}

$E F$ optimized excitation frequency, $E W$ optimized excitation wavelength, $O W$ optimal wavelength (calculated with Eq. 13), $R F$ resonance frequency

adjusted piezoceramic sensors and show a phase shift due to the different geometric dimensions.

In what follows, explanations are given regarding the meaning and the relevance of the calculated results taking into account the knowledge given in Sect. 4. Table 7 contains the optimized frequencies (EF) and their 


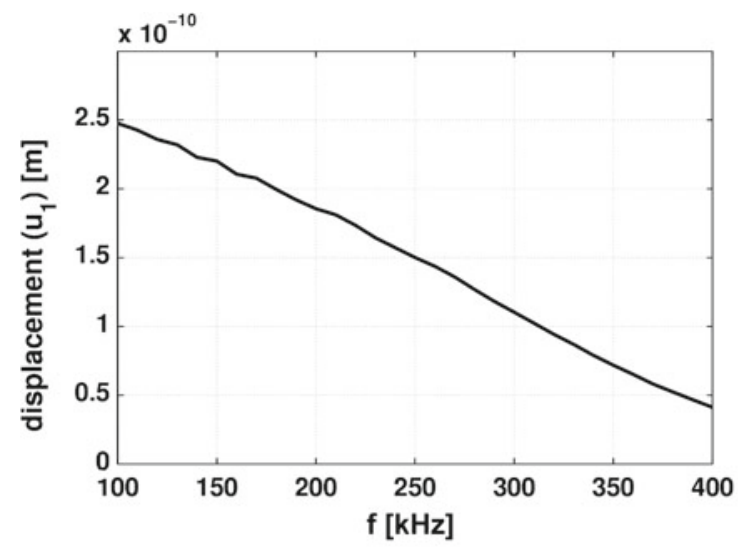

(a)

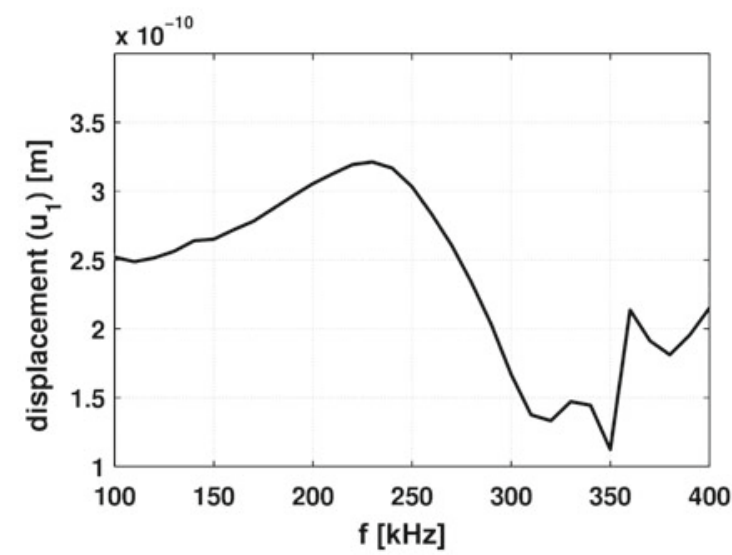

(c)

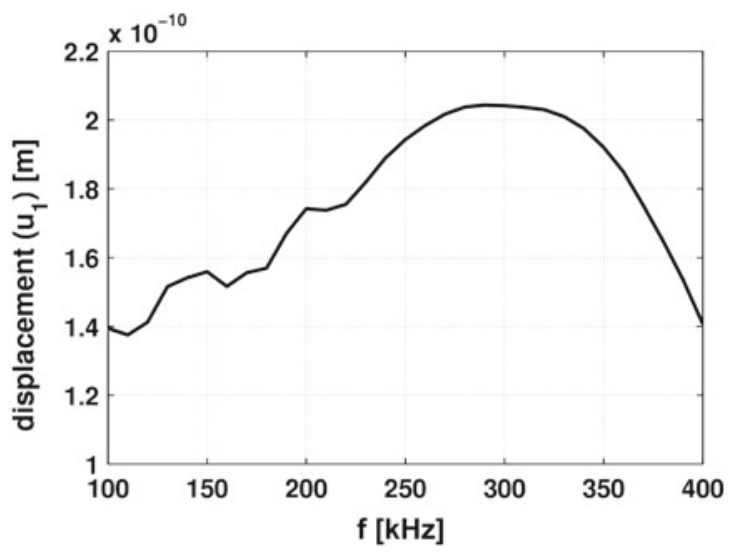

(b)

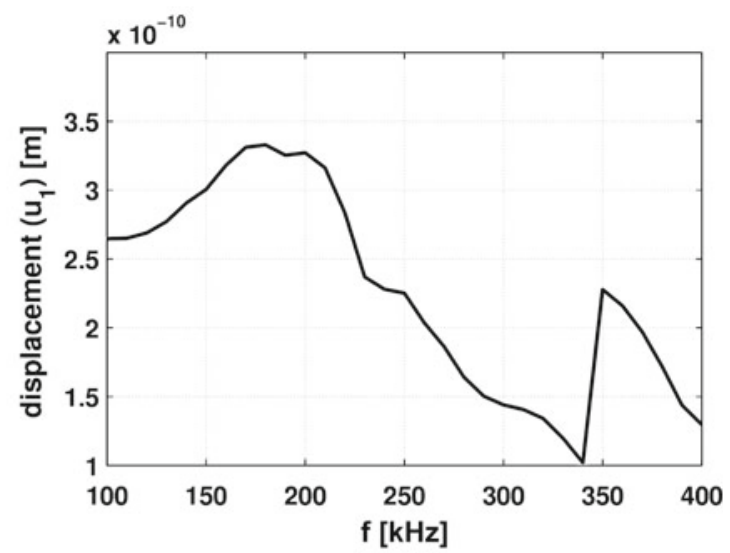

(d)

Fig. $14 u_{1}$ displacement for different excitation frequencies. a MOD1, b MOD2, c MOD3, d MOD4

corresponding wavelengths (EW) taken from the dispersion diagrams of the plate models. The wavelength $(\mathrm{OW})$ is calculated by multiplying the optimized lengths from Table 6 with two to get the first optimal wavelength (see Eq. (13) for $n=0$ ). The frequency (RF) corresponds to the resonance frequencies of the actuator plate system calculated in the range $100-400 \mathrm{kHz}$ (see Fig. 14a-d).

First, we investigate whether Eq. (13) holds for MOD1MOD4. As can be seen in Table 7, model 1 (MOD1) and model 3 (MOD3) agree quite well with the predicted solution, EW is approximately equal to $\mathrm{OW}$. The solutions of model 2 (MOD2) and model 4 (MOD4) are not consistent with the expectation. The length of the sensor of model 2 and 4 does not tend to the calculated optimal length. Hence, other effects must be more dominant. As we pointed out in Sect. 4 the resonance strongly effects the excitation of the Lamb waves. In resonance, the received signal amplitude increases significantly. All models except model 1 converge to a frequency near the resonance. For model 1 no resonance frequency exist within the give frequency range. Figure 14a shows lower amplitudes for higher frequencies. The optimal wavelength (OW) given by Eq. (13) coincides with the optimized excitation wavelength (EW). The question that arises here is why does the excitation frequency (EF) not tend to $100 \mathrm{kHz}$, where higher voltage amplitudes can be reached according to the results displayed in Fig. 14a. To fulfill Eq. (13) which results in an elevation of the received voltage signal higher excitation frequencies are favorable. Because we can infer from Eq. (8) that a greater length of the sensor increases its capacitance and thus decreases the voltage output. Lower excitation frequencies lead to higher wavelengths and hence a longer sensor length is needed to fulfill Eq. (13). For this example problem, it can be deduced that the reduction of the signal amplitude due to a longer sensor is more pronounced as the gain achieved by a lower frequency. For the models $2-4$, the optimized results show no distinct trend towards the optimal wavelength (OW). Models 2 and 4 tend to lower sensor lengths, whereas model 3 satisfies Eq. (13). As mentioned before, a longer sensor results in a higher capacitance and a reduction of the voltage. It seems that 
the both effects (fulfilling Eq. (13) in contrast to a higher capacitance) compensate each other.

From the first results, it can be seen that in general the impact of the resonance, if a resonance frequency is within the given frequency range, is more dominant than the other introduced effects. Therefore, the wavelength is determined by the eigenfrequency of the actuator. If the wavelength of the sensed Lamb wave is too long, the positive effect of the length adaption of the senor according to Eq. (13) is compensated by the negative effect of an increasing capacitance. Since for the first model (MOD1) no resonance can be found within the given frequency domain, the excitation frequency is adjusted in a way such that the wavelength is as short as possible and, additionally, the optimal wavelength is reached. More detailed investigations to quantify the impact of the different effects on the voltage output are necessary.

The thickness of the sensor tends to the upper bound of the parameter range for all four models. This can be explained by Eq. (8). Higher thicknesses result in a lower capacitance and a higher voltage output at the sensor.

\section{Conclusions}

In this paper, the influence of several parameters (resonance, adhesive layer thickness and Young's modulus) on the actuator-sensor system have been investigated. Depending on the excitation frequency and adhesive layer properties either the shear lag effect or the resonances influence the amplitude of the emitted wave primarily. The shear lag effect becomes negligible for higher frequencies, because the amplitude loss due to the shear lag effect is superposed by the resonance effect. The adhesive layer properties (thickness, Young's modulus) shift the resonance to higher or lower frequencies. To reach a higher sensitivity of the sensor, an optimization of the sensor geometry (length, thickness) as well as the excitation frequency is executed. The converged solutions show that the frequencies tend to the resonance region. If the wavelengths of such excitation frequencies are short enough the length of the sensors tend to the optimal length, which are calculated by Eq. (13). The optimized sensor geometry takes dimensions which decrease the capacitance of the sensor (Eq. 8). For greater sensor lengths, the capacitance increases resulting in a reduced voltage amplitude. A variation of the capacitance influences the received signal stronger than the optimal sensor length predicted by Eq. (13). Since the reduction of the capacitance results in a higher voltage output, the thickness of the sensor increases for all models under investigation. Thus, an optimal sensitivity of the actuatorsensor system can be achieved using the resonance frequency of the actuator and a small capacitance of the sensor.
In structural health monitoring applications, the wavelength necessary to detect damages that are critical for the structural integrity is a priori known for specific applications. Using the dispersion diagrams, it is possible to determine the frequency which corresponds to this wavelength. Since an actuator should be operating near a resonance frequency, the dimensions have to be designed to coincide with the frequency obtained using the dispersion diagram for the specific material. After the identification of the geometry of the actuator, the sensor design has to be adjusted to meet the afore-mentioned criteria for a good signal reception.

Acknowledgments The authors like to thank the German Research Foundation (DFG) and all partners for their support (GA 480/13-1).

\section{References}

1. Bäck, T.: Evolutionary Algorithms in Theory and Practice: Evolution Strategies, Evolutionary Programming, Genetic Algorithms. Oxford University Press, Oxford (1996)

2. Bartoli, I., di Scalea, F.L., Fateh, M., Viola, E.: Modeling guided wave propagation with application to the long-range defect detection in railroad tracks. NDT\&E Int. 38, 325-334 (2005)

3. Bhalla, S., Soh, C.K.: Electromechanical impedance modeling for adhesively bonded piezo-transducers. J. Intell. Mater. Syst. Struct. 15, 955-972 (2004)

4. Bohn, N.: Ein Beitrag zur Weiterentwicklung von Evolutionsstrategien für die virtuelle Produktentwicklung. $\mathrm{PhD}$ thesis, Otto-von-Guericke-University, Magdeburg (2008)

5. Bohn, N., Gabbert, U.: Evolution strategies with line search for structural optimization. In: 8th World Congress on Structural and Multidisciplinary Optimization (2009)

6. Boller, C., Staszewski, W., Tomlinson, G.: Health Monitoring of Aerospace Structures. Wiley, New York (2004)

7. Brockmann, T.H.: Theory of Adaptive Fiber Composites: From Piezoelectric Material Behavior to Dynamics of Rotating Structures. Springer, Berlin (2009)

8. Calomfirescu, M.: Lamb waves for structural health monitoring in viscoelastic composite materials. $\mathrm{PhD}$ thesis, University of Bremen (2008)

9. Ciang, C.C., Lee, J.R., Bang, H.J.: Structural health monitoring for a wind turbine system: a review of damage detection methods. Meas. Sci. Technol. 19, 1-20 (2008)

10. Crawley, E.F., de Luis, J.: Use of piezoelectric actuators as elements of intelligent structures. AIAA J. 25, 1373-1385 (1987)

11. von Ende, S., Lammering, R.: Investigations on piezoelectrically induced Lamb wave generation and propagation. Smart Mater. Struct. 16, 1802-1809 (2007)

12. Giurgiutiu, V.: Structural Health Monitoring with Piezoelectric Wafer Active Sensors. Academic Press (Elsevier), New York (2008)

13. Ha, S.: Modeling Lamb wave propagation induced by adhesively bonded PZTs on thin plates. PhD thesis, Stanford University, California, USA (2009)

14. Ha, S.: Adhesive interface layer effects in PZT-induced Lamb wave propagation. Smart Mater. Struct. 19, 1-9 (2010)

15. Ha, S., Mittal, A., Lonkar, K., Chang, F.K.: Adhesive layer effects on temperature-sensitive Lamb waves induced by surfacemounted PZT actuators. In: Structural Health Monitoring 2009: From System Integration to Autonomous Systems (2009) 
16. Huang, H., Pamphile, T., Derriso, M.: The effect of actuator bending on Lamb wave displacement fields generated by a piezoelectric patch. Smart Mater. Struct. 17, 1-13 (2008)

17. Kessler, S., Spearing, M., Atallab, M.: In-situ damage detection of composites structures using Lamb wave methods. In: Proceedings of the First European Workshop on Structural Health Monitoring, 10-12 July 2002, Paris, France (2002)

18. Lamb, H.: On waves in an elastic plate. Proc. R. Soc. Lond. Ser. A 93, 114-128 (1917)

19. Marinkovic, D.: A new finite composit shell element for piezoelectric active structures. PhD thesis, Otto-von-Guericke-University, Magdeburg (2007)

20. Pohl, J., Willberg, C., Gabbert, U., Mook, G.: Theoretical analysis and experimental determination of the dynamic behaviour of piezoceramic actuators for SHM. Exp. Mech. 52, 429-438 (2012)

21. Rechenberg, I.: Evolutionsstrategie '94. Frommann-Holzboog, Germany (1994)

22. Schwefel, H.P.: Evolutions and Optimum Seeking. Wiley, New York (1995)
23. Sirohi, J., Chopra, I.: Fundamental understanding of piezoelectric strain sensors. J. Intell. Mater. Syst. Struct. 11, 246-257 (2000)

24. Su, Z., Ye, L., Lu, Y.: Guided Lamb waves for identification of damage in composite structures: a review. J. Sound Vib. 295, 753-780 (2006)

25. Viktorov I., A.: Rayleigh and Lamb Waves. Plenum Press, New York (1967)

26. Willberg, C., Vivar-Perez, J.M., Ahmad, Z., Gabbert, U.: Simulation of piezoelectric induced Lamb wave motion in plates. In: Proceedings of the 7th International Workshop on Structural Health Monitoring 2009: From System Integration to Autonomous Systems, pp 2299-2307 (2009)

27. Wolf, F.: Präzisionsmessungen des Elastizitätsmoduls von Polymeren mit Longitudinalschwingungen II. Teil: Apparatur und Ergebnisse. Colloid and Polymer Science Kolloid-Zeitschrift \& Zeitschrift für Polymere 257, 1253-1275 (1979) 